\title{
Marketing Strategic Alternatives and Recommendations in the Distilled Spirits Industry-Sky Vodka
}

\author{
Etienne Musonera, PhD \\ Associate Professor of Marketing \\ Eugene Stetson School of Business and Economics \\ Mercer University-Atlanta \\ Atlanta, GA \\ Anand Pore, PhD \\ Assistant Professor of Management \\ Marilyn Davies College of Business, B467 \\ University of Houston-Downtown \\ Houston, TX
}

\begin{abstract}
SKYY Vodka began in 1992 as a tiny start-up in San Francisco and based on the vision of entrepreneur Maurice Kanbar and his dream to make exceptionally smooth vodka, SKYY is now the leading domestic premium vodka in the United States. SKYY Vodka was originally sold by SKYY Spirits LLC, which was later acquired by Campari Group - later renamed Campari America. Currently, it is now the U.S. marketing arm of Campari, and markets other Campari products in addition to the vodka line.Over the last few years, SKYY Vodka has invested a series of entertainment-based marketing campaigns designed to craft a "glamorous and sexy" brand image. In this paper, we performed a complete case analysis and addressed Sky Vodka's problems found in the situation analysis from its inception to now. We also used Michael Porter's Five Forces to conduct industry analysis and we provided strategic alternatives marketing strategies and recommendations.
\end{abstract}

Keywords: Sky Vodka, Michael's Five Forces, Marketing Strategies

\section{Introduction}

SKYY Vodka began in 1992 as a tiny start-up in San Francisco. Based on the vision of entrepreneur Maurice Kanbar and his dream to make "exceptionally smooth vodka," SKYY is now the leading domestic premium vodka in the United States. SKYY Vodka was originally sold by SKYY Spirits LLC, which was later acquired by Campari Group - later renamed Campari America. It is now the U.S. marketing arm of Campari and markets other Campari products in addition to the vodka line. SKYY Vodka prides itself on its state-of-the-art quadruple distillation process, designed to eliminate toxins - resulting in a cleaner vodka that reduces the occurrence of hangovers. SKYY Vodka relies heavily on entertainment-based marketing campaigns to promote its brand and connect with consumers. Having started with product placement in movies, they developed the campaign across all marketing platform, from advertising to the creation of "cinematic cocktail moments" in short films to pointof-purchase displays. Over the last few years, SKYY Vodka has invested a series of entertainment-based marketing campaigns designed to craft a "glamorous and sexy" brand image. The results of their marketing campaigns have been outstanding: SKYY's brand awareness and product trials have doubled, and advertising awareness has tripled. Because the brand has strong roots in film, it gives the vodka a cultural anchor and heritage in modern society. SKYY Vodka's primary problem consists of competition in the market: Many other vodka brands are jumping on the Hollywood placement bandwagon, making it increasingly difficult to get results. With other vodka brands pursuing this form of advertising, SKYY brand risks getting lost in the avalanche of product placement. Unless their message is extremely relevant, consumers may not remember which brand is associated with what. This presents a huge responsibility to SKYY Vodka to create a marketing campaign that is unique and original, not following any traditional liquor advertising clichés. By continuing to focus on the brand's image, SKYY will be able to differentiate itself from competitors and continue to enjoy its reputation as the leading domestic premium vodka in the United States. 


\section{Situational Analysis}

1.1. Economic Conditions and Trends: Overall, the future looks positive for the distilled spirits industry particularly in the vodka category. According to the latest full year data, the global spirits market expanded by $11 \%$ in volume between 2012 and 2013. Despite the weak global economy, premium and super-premium vodka brands are growing due to a focus on entertainment-based marketing campaigns and aspirational consumers. Social media provides an excellent opportunity for alcohol retailers to connect with consumers. There are innovative social media campaigns currently being used by competitors, but interaction doesn't necessarily lead to engagement. However, the industry experienced 10\% growth in sales value between 2012 and 2013.

1.2. Cultural and Social Values and Trends: U.S. consumers are increasingly concerned about the effects of excessive drinking on both health and personal appearance. Due to this trend, 'Skinny' or low-calorie, premixed cocktails are growing rapidly in the ready-to-drink (RTD) category and young women are the demographic driving RTD growth in the U.S. Research shows that women are more likely than men to consider 'Skinny' vodka brands and be concerned about health and personal appearance. Alongside the skinny trend, low-alcohol cocktails, wines, and RTD variants are taking off in the United States. Product innovation in spirits continues to focus on a proliferation of flavors. In the U.S., the flavored vodka category expanded by $25 \%$ between 2012 and 2013, making it the fastest growing segment of the category. Vodka brands are making strides in finding ways to exploit the opportunity that social media represents. Brands are changing the tone and content of advertising to better resonate with consumers. Major companies in the industry have launched innovative responsible drinking campaigns.

1.3. Summary of Environmental Opportunities and Threats: SKYY has a variety of environmental opportunities.

Consumes of vodka are increasingly desiring the success and self-actualization that premium brands symbolize. Spirits premiumization is occurring because consumers are increasingly searching for value for the money they spend. Premiumization is a journey for the consumer - as they progress in life, they will seek to satisfy higher needs, rather than basic needs. Research indicates that $88 \%$ of consumers consider spirits an affordable luxury. To consumers, "affordable luxury" means quality and value. SKYY Vodka responded to this demand by introducing their ultra-premium vodka - SKYY 90.

\section{Industry}

2.1. Classification and Definition of Industry:SKYY Vodka is a domestic leader in the premium vodka market and is very competitive. Compared to the distilled spirits industry, the vodka category is now one of the most popular styles of liquor. The vodka industry has been characterized by marketers as a Cash Cow: it is highly profitable due to high market share in the distilled spirits industry, has low growth potential, many competitors, and difficult to attract new customers.

2.2. Analysis of Existing Competitors: There are many producers of vodka, including the big-name and countless boutique brands that continue to enter the market. This list includes the major brands of vodka that almost everyone recognizes when ordering cocktails. These vodka brands are direct competitors of SKYY Vodka and can be found on nearly every bar and liquor shelf. They are also ideal for virtually any vodka cocktail you wish to mix.

2.2.1. SKYY Vodka: SKYY Vodka is the number-one domestic premium vodka and the fifth biggest premium vodka globally. SKYY's unique, state-of-the-art process of distillation and filtration yields a quality vodka with a smooth taste. SKYY's iconic cobalt blue bottle and marketing campaigns have made the SKYY brand synonymous with quality, sophistication, and style.

2.2.2. Absolut Vodka: Absolut Vodka received most of its notoriety from innovative advertising campaigns that depict bottles of their product in various graphically enhanced situations. In addition to their innovation in flavors, their campaign has worked extremely well over the years. Their line of flavored vodkas is unique, but the quality and taste of their product has received mixed reviews.

2.2.3. Grey Goose: Grey Goose has been heralded as a favorite "go to" vodkas, because of its simplicity and consistency. Grey Goose has a reputation of being one of the smoothest and most mixable vodkas and is a staple among vodka drinkers. Grey Goose also produces a line of flavored vodkas. The most popular being La Poire, pear-flavored vodka, and is used in a variety of signature cocktails. 
2.2.4.Van Gogh: When it comes to premium vodkas, Van Gogh is touted as one of the best among its competitors and consistently tops the "favorite brands" list. It began as a Dutch gin distillery and is now a benchmark in vodka due to its smooth, clean, and refreshing taste. They are seeing much success with their Van Gogh Blue, a triple wheat vodka that has a profoundly smooth and flavorful taste.

2.2.5. Ketel One: The Ketel One brand thrives on the legacy of the Nolet family of Dutch distillers, which are now in the $10^{\text {th }}$ generation. Their two flavored vodkas (Citroen and Oranje) are delicately flavored and are commonly used by bartenders to make Vodka Tonics and Martinis.

2.2.6. Belvedere: Belvedere is a Polish vodka that comes in an elegant frosted bottle that looks great on display in bars. Their original, clear bottling is the flagship of the brand and is mostly used for high-class cocktails. It has a unique taste that is derived from the use of rye, giving it a distinctive flavor. The brand is not as well known for the rest of their product line, however.They have been experimenting with new flavors such as pink grapefruit, black raspberry, orange, citrus, ginger, almond, cinnamon, and jasmine.

2.2.7. Stolichnaya: Stolichnaya or simply "Stoli", is an everyday premium vodka that is used mostly for mixing cocktails. This is touted as being one of the best Russian vodkas in the world. Most noticeably is the fact that this brand has flavors with a decidedly Russian flare to their names, such as; Peachik, Strasberi, or Oranj. All flavors have a unique spelling, which is a definite distinguishing mark.

2.3. Analysis of Potential New Entrants: Successful new entrants are finding that established players in the distilled spirits industry are interested in gaining the cachet that comes from a smaller, niche brand. Larger distilleries have already begun to buy smaller competitors. The largest four players within the spirits industry - Diageo, Brown-Forman, Beam, and Pernod Ricard SA have also acquired smaller additional brands and expanded their total market share to 63.2 percent over the past five years. Therefore, new entrants are being bought out before they have an opportunity to gain traction in the industry.

2.4. Analysis of Substitute Products: The threat of substitutes for premium vodka is low, as consumer would not likely substitute a premium vodka for a lower-end or "well" vodka due to the importance of brand recognition. While many consumers' preferences will not change, the frequency of their purchases could decline. Premium brands like SKYY would suffer the most from decreased consumption at bars and restaurants, where consumers may opt for cheaper brands or reduce their entertainment costs.

2.5. Analysis of Suppliers: Gruppo Campari, the Italian drinks group that owns SKYY Vodka, has signed an agreement with a larger distributor in Australia for the distribution of the SKYY Vodka brand. Under the terms of the deal, SKYY Vodka will be distributed throughout Australia, which will secure a greater share of the Australian premium branded spirit market. At more than 500,000 cases sold per annum, vodka is Australia's fastest growing spirit category, with consumer demand for known international vodka brands driving this growth. Despite its Italian owner, SKYY Vodka is not widely known in Europe. However, SKYY's reputation in the U.S. vodka market has already led to a cult following in Australia.

2.6. Analysis of Buyers: Vodka is America's top-selling spirit accounting for nearly a third of the liquor market and half of its growth. Demand for premium vodka has continued to grow despite an economic downturn in the U.S. Vodka sales in the U.S. amounts to $\$ 13,403$ million in 2018 and expected to grow annually by $2.8 \%$ (www.statica.com). The U.S. is the primary market for premium vodkas despite the sluggish economy. Vodka is an inherently profitable category for the SKYY brand because vodka is a product that can be made and sold quickly, which means that the demand from buyers can be satisfied almost immediately.

2.7. Summary of Industry Opportunities and Threats: SKYY Vodka has a few opportunities that it would be wise to address. First, there are the emerging economies of Brazil, Russia, India, and China (the BRICs). These countries represent a newly advanced economic development and massive population. SKYY should seek distribution in all four countries and develop a cutting-edge marketing strategy to engage the consumers. Next, there is a recent shift in consumer preferences toward premium-priced spirits. SKYY has already recognized this trend and has begun marketing campaigns for its ultra-premium vodka - SKYY 90. Lastly, the cocktail culture continues to thrive throughout the United States, which presents an opportunity for SKYY to differentiate themselves through their SKYY Infusions product, which has gained significant popularity among consumers. SKYY Vodka is faced with several threats. First, there is heavy competition within the spirits market. It has become increasingly difficult for brands to differentiate themselves because of the mass product placement advertising. SKYY will need to continue to employ innovative marketing strategies that will communicate a clear message to consumers to stay ahead of its competitors. Next, there is emerging competition among private labels within local markets. 
Because of the low barriers to entry and the growing number of private label distilleries present, new entrants are entering the market at a rapid rate. Lastly, competitive pricing is a primary driver of consumer loyalty. Other premium vodka brands are using competitive pricing to lure consumers. SKYY Vodka is no longer the only affordable premium vodka on the market.

\section{Organization}

3.1. Objectives and Constraints: SKYY Vodka's singular mission, from the beginning, was to create an "exceptionally smooth vodka." Since its creation in 1992, it has sought to improve the taste and experience of vodka by removing all impurities that distract from its true flavor. SKYY prides itself on its state-of-the-art quadruple distillation and triple filtration process. The investments they have made into their distilleries is proof of their commitment to producing a quality product. Since SKYY Vodka is an adult beverage, it cannot be marketed to minors. Before you can enter their website, you must enter your birthday. Obviously, this alone will not prevent minors from accessing the site if they want to, but it speaks to the company's willingness to be responsible in the advertising of their product. SKYY is also constrained by its distribution. SKYY Vodka is distributed internationally, however its SKYY Infusions and SKYY 90 brands only have distribution in the United States and Canada.

3.2. Financial Condition: SKYY Vodka is performing well, financially. Net sales increased by $\$ 66.6$ million between 2011 and 2012, although net profit dropped by $\$ 2.5$ million due to a rise in debt (www.skyy.com).

3.3. Management Philosophy: SKYY's philosophy is to provide a premium vodka with the purest, smoothest taste with less impurities than any other vodka. SKYY further seeks differentiation in the premium vodka market by associating its brand with style and sophistication - making it the spirit-of-choice among young professionals.

3.4. Organizational Structure: SKYY Spirits, LLC is an American spirits maker. It was launched in 1992 and founded by Maurice Kanbar in San Francisco. Its majority shareholder is the Campari Group. SKYY Spirits manages Campari's portfolio in the U.S. SKYY Spirits is the sole importer of Campari into the U.S. and the international distribution arm of Campari. In 2001, Campari Group became controlling shareholder of SKYY Spirits with 58.9\% stock ownership. In 2005, Campari acquired a further $30.1 \%$ share in SKYY Spirits, increasing its total share to $89 \%$ (www.skyy.com).

3.5. Organizational Culture: SKYY Vodka is a premium vodka brand. Through its hip culture, it seeks to change the consumer's perception of the brand by repositioning it as a "step up" for the young professional. Further, SKYY aims to increase trial among this new target market and to increase purchase intent through product differentiation. Ultimately, SKYY strives to increase sales and awareness and gain market share in the vodka category.

3.6. Summary of Firm Strengths and Weaknesses: SKYY Vodka is the number one "domestically" made premium vodka. It is quadruple distilled, high quality product that significantly improves taste and reduces hangovers. SKYY is a well-known brand that is competitively priced among other premium vodkas. While SKYY brand has a good position in the industry, premium vodka brands such as Grey Goose are increasing their sales due to the growth of premium and super-premium vodka markets. SKYY should continue to differentiate its products from competitors and may also want to re-position itself. One of the strengths is the pricing: for a premium vodka, it is affordable for most people, including college students and young professionals. By building emotional ties to these strengths, SKYY will be able to position the product as "professional yet attainable" vodka, which is ideal for young professionals seeking an enjoyable social life.

\section{Marketing Strategy}

Objective and Constraints: SKYY Vodka has a marketing strategy that relies more on entertainment-based marketing than traditional commercial advertising. They want consumers to view their product as a good value for a premium vodka brand, thus creating brand loyalty. They advertise heavily in movies and films, thus making an icon out of the SKYY brand.

4.1. Analysis of Sales, Profits, and Market Share: Net sales for the year 2018 were $\$ 1,340.8$ million, an overall increase of $5.2 \%$, due to organic growth of $2.8 \%$, a positive exchange rate effect of $2.2 \%$ and a marginal external growth effect of $0.3 \%$. Gross profit was $\$ 769.5$ million, up $4.7 \%$ compared with the previous year. 
As a proportion of sales, it was $57.4 \%$, compared with $57.7 \%$ in 2011 , due to the greater incidence of the cost of goods sold in the spirits category, SKYY Vodka performed very well, benefiting from the introduction of their SKYY Infusions brand.

4.2. Analysis of Target Market: SKYY Vodka is known as a "brand high above others". It is stylish and fashionable. It targets young professionals and studies also show that $40 \%$ of the total sales are from underage drinkers (www.statoica.com).

4.3. Analysis of Marketing Mix Variables: A 375ML bottle of SKYY Vodka usually averages between $\$ 15$ to $\$ 25$ in the United States. Campari Group distributes SKYY Vodka nationally and in more than 100 markets outside of the United States. SKYY Spirits also distributes outside brands. SKYY is the exclusive US importer for Campari, Cutty Sark Scotch Whisky, Cinzano vermouths, and other spirits. As of May 20, 2012, SKYY Vodka sales reached 61.2 million dollars, about 6.4 points increase from the prior year.

4.4. Summary of Marketing Strategies Strengths and Weaknesses: The Alcohol Industry has been a thriving part of global culture for decades, vital to this nation's economy and a significant part of its history. Currently, SKYY Vodka's direct industry - distilled and blended liquors - is valued at approximately \$95 billion. Furthermore, the industry continues to grow in America, as seen in the 3.1\% increased consumption between 2003 and 2004. Vodka sales rose 4\% over the year as well. Unfortunately, global sales appear to be falling at a very similar rate, with Tequila being the only category to rise in the last 10 years. Vodka has been the most stable, losing only $0.7 \%$ of sales over the 10-year period. The Alcoholic Beverage industry is currently dominated by a few very large companies, which in turn branch off and offer a variety of subsidiaries and products. However, unlike the brewing industry where there is an emerging duopoly, the Distilled liquors industry has a wider spread of companies. As a result, each of these companies can produce the various distilled liquors - Vodka, Gin, Whiskey, Tequila, and Rum - to sell to different markets. Furthermore, the various classes and qualities of these liquors allows for a discrepancy in pricing. The current fad for businesses in the Alcohol industry has been to infuse foreign substances and flavors to improve the taste or effect of their product. For instance, emerging "PINK" Spirits are infused with caffeine and Ginseng to improve their effects. Whether this trend will remain is questionable, but SKYY has steered clear from the trend so far, their only exception being SKYY Citrus.

\section{Problems Found in Situational Analysis}

5.1. Statement of primary problem: The primary problem is the difficulty of differentiating product with others available on the market. Vodka, inherently, is sought after primarily for its purity and lack of disruptive qualities when used for cocktails and mixtures. This means that the product only excels when it is unnoticed.(i) Evidence of problem: In an environment that can include all kinds of fruit juice, syrup, sweet and sour mixture, and even straight-up granulated sugar, an average consumer probably won't be able to distinguish vodka A from vodka B based on taste alone. This can be seen when the consumer asks for a mixed drink and doesn't specify the brand of vodka used.(ii) Effects of problem: This problem leads to a false lack of demand by consumers. This is false in that it can also be attributed to other brands but can still affect vendor and restaurant demand for SKYY. If any vodka product will suffice, or if the bar manager wants to make the shelves look pretty with a wide range of bottles that mostly won't be used, this can be classified as a failure.

5.2. Statement of secondary problem: Given the heated nature of the American alcohol industry in general and vodka in particular, SKYY faces a threats from all sides in the form of new entrants and substitute products. $(i)$ Evidence of problem: A sharp increase in the offerings of flavored vodkas can replace standard vodka as a mixing platform while new labels that are purchased by other big-name companies to provide an infusion of fresh thinking and fresh products are prime examples.(ii) Effects of problem: Clubs and bars that are looking to revitalize their offerings will look to add unknown products to their line-up and younger drinkers might attempt to differentiate themselves from older drinkers by adamantly keeping away from what their parents enjoyed. Wine pairings are more commonly offered with meals which displaces a mixed drink before or after food is served.

\section{Strategic alternatives for solving problems}

6.1. Description of strategic alternative 1: To better distinguish SKYY from other vodkas, information needs to be made available to consumers on why they should choose it over other brands. Distillery tours or an 
intensified mixed-drink recipe campaign would impart such knowledge on customers to develop an appreciation for the product.

(i) Benefits of alternative 1: Like with beer breweries, vodka consumers can use the opportunity to see their favored beverage being made while getting to try fresh samples and socializing with friends and other enthusiasts. A more active mixed-drink campaign, involving visits to bars and clubs nationwide as well as the traditional television commercials and package store stands can be used to display new ways to utilize SKYY.(ii) Costs of alternative 1: The costs for modifying the distillery where SKYY's vodka is made would be a significant investment up front, but recouping loses with admission cost and souvenir sales could help to offset that. Travel cost for employees to bars and clubs is insignificant by comparison to getting the products to the location.

6.2. Description of strategic alternative 2:Increasing SKYY's presence in the flavored vodka market might be their next logical move. Given the rise of consumption on such products, SKYY hasn't fully taken advantage of this segment. With only an $80 \%$ growth in the flavored market, SKYY has room to grow.(i) Benefits of alternative 2: An increased breadth of product line with increased awareness of the rest of SKYY's offerings when a consumer orders a flavored drink, would be beneficial.This will also allow SKYY to help separate itself from other vodka brands should it provide more unique or exciting flavors. (ii) Costs of alternative 2: There are increased costs with $R \& D$ that always comes with the science of creating new flavors. There is also the challenge of figuring out what consumers will like in conjunction with not mimicking existing rival flavors and look like unoriginal copycat. Bottle design, release options, and new mixed drinks ideas all add to the costs.

\section{Selection of Strategic Alternative and Implementation}

7.1. Statement of selected strategy: The strategy with the greatest sense of return is undoubtedly the attempt to educate consumers on the differences and benefits of SKYY brand. This includes an on-the-scene advertising campaign with bars and clubs set as the destination. Free samples of products and efforts to inform consumers in the process of consuming will also be included.

7.2. Justification for selection of strategy: The grassroots movement is something that has appeal with most consumers, specifically younger people that use social media to indicate their whereabouts and activities as something unusual and exciting. This will set SKYY apart from competitors with an "anti-corporate" attitude indicating that SKYY has the interest of the individual customer at heart and simply content with just putting an impersonal commercial on the television during a sports event.

7.3. Description of implementation of strategy: The method of implementation is simple and straight-forward. On a Friday or Saturday night, at any venue that verifies age at the door and sees high volumes of alcoholconsuming customers, a SKYY promotions group would show up to mingle with the crowd and offer samples of different SKYY products. Consumers that show interest in a specific type of mixed drink would be offered information on how the drink is made with SKYY vodka as the base. Reinforcing how SKYY is meticulously crafted to produce a superior product will impress upon the minds of potential alcohol buyers a dedication to the product that translates to a better time for them. Encouragement for patrons to share on social media the fun they're having will also be in effect to further spread awareness of the events.After this initial period, filmed footage of these small events can be utilized for a commercial campaign showcasing these efforts and further adding awareness to unreached consumers.

\section{Summary\& Conclusion}

It's apparent that the alcohol market is changing from its more traditional design, but the fact remains that the United States presents with the most appealing geographic option. New entrants, substitutions, and sociological shifts all present a daunting challenge but SKYY can capitalize on this opportunity by distinguishing itself from the rest of the segment with a fresh presentation on what this recreational drink can offer. The company's strength has always been its innovation and creativity in business. It has been ingrained in the company's culture from inception and that's why it remains one of the top five best-selling spirit brands worldwide. As the company vision and mission state, its success lies in Skyy's ability to create trends, to add an additional dimension of style and sophistication with its product. Its future success will be determined by its ability to rebrand itself as the number one premium spirit worldwide. Maintaining its market share in the United States while branching out to untapped markets globally should remain true to the company's passion, as an innovator in brand marketing, a partner in social awareness, and overall responsible corporate citizen. 


\section{References}

Brown, E. (1998). Will Uncle Maurice Let Go? Forbes, September 7, p. 92.

Bruss, J. (2002). Compari Takes to the SKYY. Beverage Industry, January, p. 8.

Demeter Group Investment Bank. (2014). State of the Spirits Industry 2013." (2013): 1-15. 2013. Web. 19 Sept.

Ellett, J. (2014). Absolut Vodka Launches New Campaign, Breaks with The Past. Forbes.

Forbes Magazine, 09 Sept. 2103. Web. 22 Sept. 2014.

<http://www.forbes.com/sites/johnellett/2013/09/09/absolut-vodka-launches-new-campaign-breaks-with-thepast/>.

Gordon, D. (2001). Curing Fuzzy Heads and Fuzzy Sweaters. Toronto Star, February 18.

Gruppo Campari. (2014). Acquisition of SKYY Spirits, LLC." (n.d.): n. page. 13 Dec. 2001. Web. 19 Sept. 2014.

Hamilton, William L., "Pie-in-the-Sky Spirits," New York Times, March 30, 2003, p. 9.

Hein, K. (2002). SKYY Sets the Stage in Sultry Cinematic Scenes. Brandweek, June 17. p. 8.

Hochstein, M. (1995). Nation's Restaurant News. February 13, p. 45.

Howard, T. (1999). Marketers of the Next Generation: Teresa Zepeda. Brandweek, November 8, p. 38.

https://www.skyy.com/about/

https://drizly.com/skyy-vodka/p4661

https://www.camparigroup.com/en/brands/vodka/skyy-vodka

http://campariamerica.com/brands/skyy-vodka/

https://vodkabuzz.com/vodkas/skyy/

http://www.liquorlive.com/

https://www.totalwine.com/vodka/flavored-vodka/cranberry/skyy

https://distiller.com/spirits/skyy-vodka

http://www.businesswire.com/news/home/20151102005195/en/

Kanbar, M. (2000). Beverage Industry, June, p. 78.

Shapiro, E. (1994). Marketing: 'Hangover-free' Vodka Makes Some Queasy. Wall Street Journal, October 31, p. B1.

www.statica.com

Sinton, P. (2001). Campari Takes Bigger Slice of SKYY. San Francisco Chronicle, December 14, p. B1. 